\title{
RF CONTROL SYSTEM FOR SUPERCONDUCTING PROTON LINAC
}

\author{
B.I.Bondarev, A.P.Durkin, Yu.D.Ivanov, I.V.Shumakov, N.I.Uksusov, \\ Moscow Radiotechnical Institute, Warshawskoe Shosse 132, 113519 Moscow, Russia
}

\begin{abstract}
$\mathrm{RF}$ control system for superconducting proton linacs is presented. The system based on maintenance of phase difference between two resonance detectors is proposed. Results of beam simulation in linac with using this system and taking into account RF field perturbation in different cavities are discussed.
\end{abstract}

\section{INTRODUCTION}

Modern designs of high power accelerators include long accelerating channel consisting of short superconducting cavities. This channel consists of several hundreds cavities and so large number leads to high sensitivity of output beam parameters to RF field phase and amplitude instabilities. Linac reliable operation is made possible with RF field phase instability limited by $0.5^{0}$ and amplitude instability by $0.5 \%$. Peculiarity of accelerating system with short cavities implies that multiple cavities are driven by one common high power klystron. In that case field stabilization in individual cavity is difficult or impractical at all. So it is desirable to use correction system of beam phase position and to investigate peculiarity of beam phase dynamic with such system. Correction method of beam center phase position based on resonance detector of beam current is presented. Beam dynamic investigation results in support of feasibility and advantages proposed control method are presented as well.

\section{CORRECTION METHOD}

Main control scheme is presented at Fig.1. One RF generator (AC) drives cavity assembly. Two values resulting from accelerating channel calculation are used: phase of equilibrium particle at the assembly input (detector position) and phase difference between output and input (detectors positions). System consists of two beam phase detector BPD1 and BPD2, amplitude control system ACS and phase control system PCS. RF signal from first cavity of the assembly is compared with base RF signal from BPD1. PCS corrects generator phase so that beam phase at BPD1 position coincides with calculated equilibrium phase. In this case there is no BPD1 signal. BPD2 presents beam phase at assembly output and PCS is adjusted to nominal calculated value, so that with nominal phase and energy of bunch center BPD2 signal is equal to zero. With phase difference variation from nominal value ACS corrects field in all cavities of this assembly so that compensate arisen discrepancy. As a result of correction phase difference between assembly input and output becomes equal to nominal value (with demanded accuracy), although fields in the cavities may be differed from its nominal values.

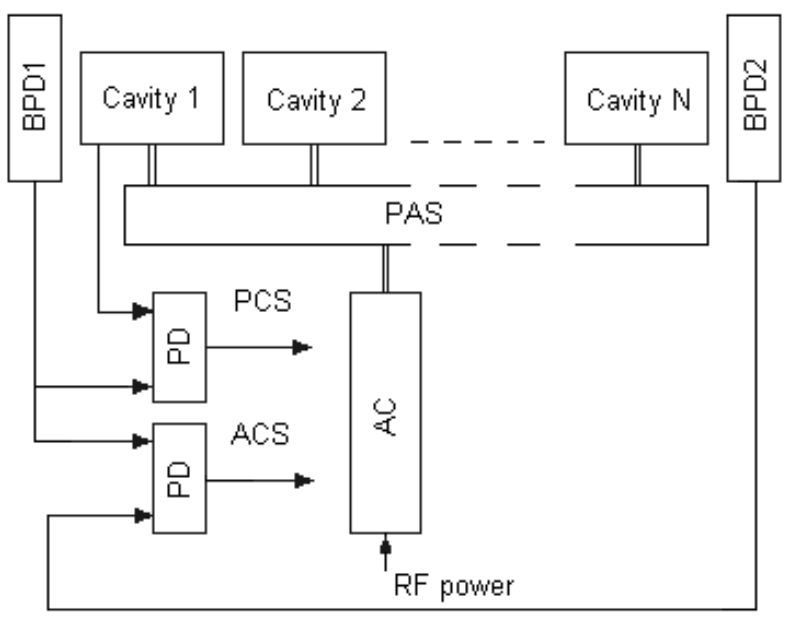

Figure 1: Control scheme. PAS - power allocation system, AC - amplification channel, BPD1, BPD2 - beam phase detectors, PD - phase detectors, PCS - phase control system, ACS - amplitude control system

\section{BEAM DYNAMICS INVESTIGATION CODE}

Computer code package for accelerating channel calculation and Monte-Carlo beam dynamic simulation was developed. It was used for checking of proposed correction method, revealing its special features and estimation of various parameter influences on final result. Code package makes it possible to chose number of cavity types (all cavities of each type have the same cell length), to determine equilibrium phase at the input and output of each cavity and group cavities driven by one RF generator as well. Initial data are as follows: number of cell in the cavity, minimal acceleration efficiency for each cavity type, RF field amplitude, distance between cavities, beam current, RF generator power for beam acceleration (useful power). Calculated channel version is simulated by Monte-Carlo method first without correction, and then with correction. Amplitude of phase errors and relative field errors are preset and $\mathrm{N}$ random realizations of accelerating channel are calculated. In each realization random additives is added to field phase and

*e-mail:lidos@aha.ru 
field amplitude. With correction procedure phase of beam center at the beginning of cavity assembly driven by one $\mathrm{RF}$ generator is returned to equilibrium value. After that the procedure of amplitude RF field variation switch on. During this procedure simultaneous increase or decrease of RF fields in each cavities of one assembly takes place until output phase of beam center became equals to calculated equilibrium phase with specified accuracy.

\section{INVESTIGATION RESULTS}

A channel that contains five-cell cavities with operating frequency $1056 \mathrm{MHz}$, accelerating rate $5 \mathrm{MeV} / \mathrm{m}$, RF field intensity inside gaps $12 \mathrm{MV} / \mathrm{m}$ and $0.5 \mathrm{~m}$ as distance between cavities was consider as base for investigations. The channel was used for $30 \mathrm{~mA}$ proton beam acceleration from energy $100 \mathrm{MeV}$ up to $1000 \mathrm{MeV}$. The total cavity number is 386 (5 types). Input beam phase width equals $30^{\circ}$ and beam is considered without energy spread. The calculations below are performed taking into account that $0.5 \mathrm{MW}$ is maximal RF power used for beam accelerating. In such case 11 cavities are powered by one power supply at the channel beginning and 6 cavities are powered at the channel end.

Beam center trajectories are shown at Figs 2 and 3 for beam with input energy increased by $0.1 \%$ regarding nominal one. At Fig.2 beam correction is switch off, at Fig. 3 it is switch on. RF field random perturbations are absent so in the case without correction we have usual phase oscillations. Correction using leads to full phase oscillation reduction.
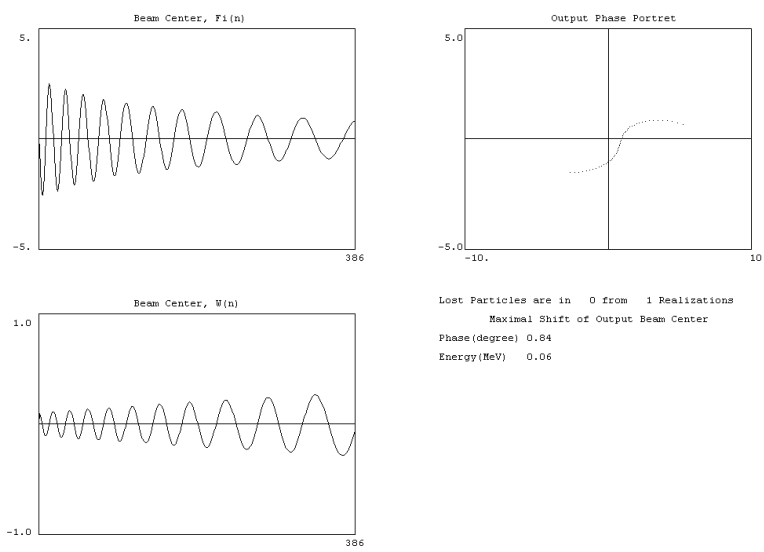

Figure 2: Beam dynamics with initial energy of 100.1 $\mathrm{MeV}$ (without correction)

At Figs 4 and 5 beam center trajectories are compared for 50 random realizations of the channel with $\delta \mathrm{E} / \mathrm{E}=1 \%$ and $\Delta \varphi=1^{0}$ as tolerances for RF amplitude and phase. At left side of the figure is shown superposition of beam center phase and energy deviations from equilibrium values for all random realizations. At right side of the figure is shown phase portrait superposition.
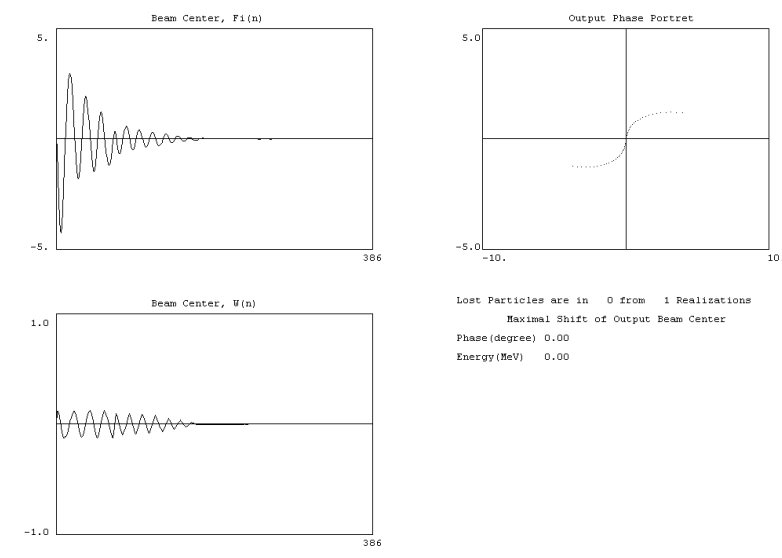

Figure 3: Beam dynamics with initial energy of 100.1 $\mathrm{MeV}$ (with correction); generator useful power of $0.5 \mathrm{MeV}, 6-11$ cavities in assembly

As can be seen correction give a possibility to reduce beam center maximal phase and energy deviations from $12^{0}$ and $4 \mathrm{MeV}$ up to $2.2^{\circ}$ and $0.17 \mathrm{M \ni B}$.
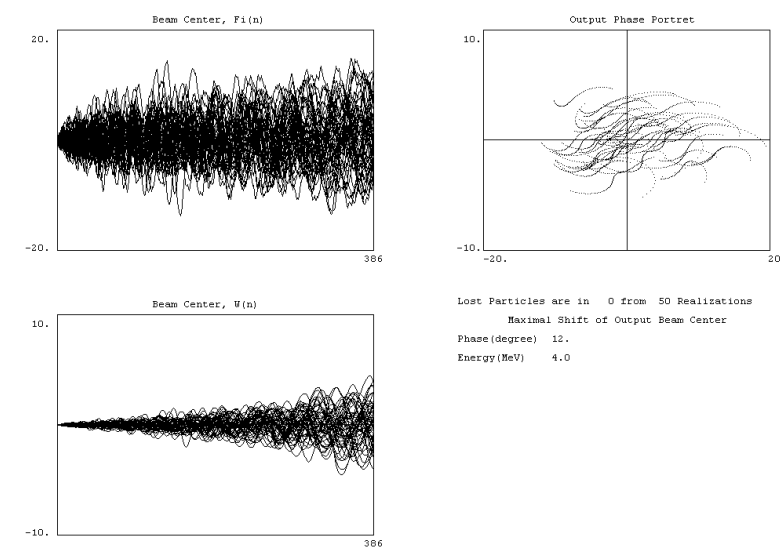

Figure 4: Fifty random realization of beam channel (without correction) $\delta \mathrm{E} / \mathrm{E}=1 \%, \Delta \varphi=1^{0}$

The proposed method efficiency depends on cavity number powered by one RF supply. The number of cavities powered by one RF supply is increased with RF supply power increasing. In this case perturbation recompense inside cavities due to RF field amplitude correction leads to beam center phase oscillation growth especially at the beginning part with short cavities. So with increasing of RF supply useful power up to $0.8 \mathrm{MW}$ (16 cavities inside one block) particle losses were observed in several random realizations via large phase oscillations. From the other side efficiency of proposed method increased sharply with decreasing of cavity number. If power of one RF supply equals $0.25 \mathrm{MW}$ (cavity number from 6 up to 4 ) and $\delta \mathrm{E} / \mathrm{E}=2 \%, \Delta \varphi=2^{0}$ maximal deviation of beam output energy is reduced from $8.7 \mathrm{MeV}$ in channel without correction up to $0.21 \mathrm{MeV}$ in channel with correction. In the last case maximal amplitude of beam center phase oscillations not exceed $7^{0}$. 

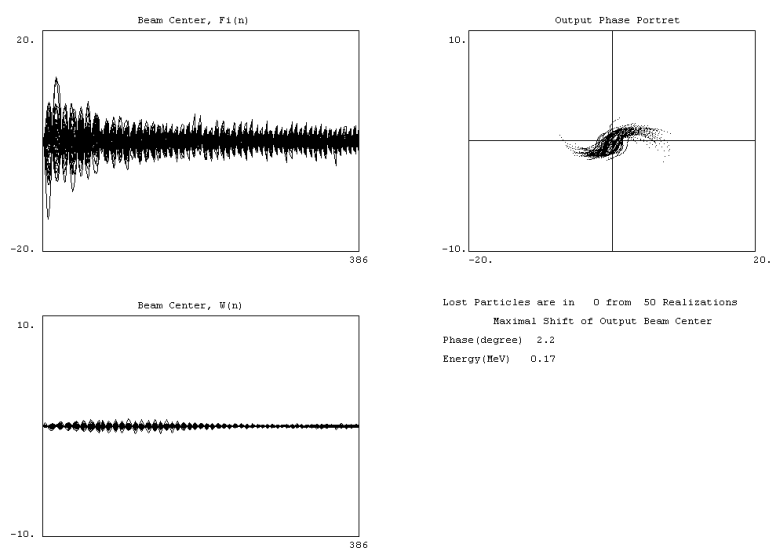

Lost Particles are in o from 50 Realizations
Maxiral shift of Output Eean Center $\begin{array}{lll}\text { Phase (degree) } & 2.2 \\ \text { Energy (KeEv) } & 0.17\end{array}$

Figure 5: Fifty random realization of beam channel (with correction); $\delta \mathrm{E} / \mathrm{E}=1 \%, \Delta \varphi=1^{0}$, generator useful power of $0.5 \mathrm{MW}, 11-6$ cavities in assembly.

In situation when power of one RF supply equals $0.5 \mathrm{MW}$ the same deviation of beam output energy was obtained with maximal amplitude of beam center phase oscillations not exceed $20^{\circ}$. Beam losses not observed even with tolerances $\delta \mathrm{E} / \mathrm{E}=5 \%, \Delta \varphi=5^{0}$ if power of one RF supply equals $0.25 \mathrm{MW}$. In this case maximal deviation of beam output energy was observed $0.47 \mathrm{MeV}$ with maximal amplitude of beam center phase oscillations not exceeds $15^{\circ}$. The change of RF field amplitude was no more then $10 \%$.

It is well known correction method based on preservation of vector sum for RF field inside cavities powered by one RF supply [1]. Proposed correction method is based on maintenance of phase width of channel part driven by one RF supply. It seems simplest in realization because maintenance accuracy is defined only by an accuracy of beam phase control.

\section{CONCLUSION}

1. The investigations confirmed that proposed correction method could be effectively used for superpower accelerators with short superconducting cavities.

2. The new correction method will be useful if cavity number not exceeds 10 .

3. The proposed correction method gives good results for output beam if cavity number not exceeds 10 in the beginning part and with $\delta \mathrm{E} / \mathrm{E}=1 \%$ and $\Delta \varphi=1^{0}$ or not exceeds 6 in the beginning part and with $\delta \mathrm{E} / \mathrm{E}=5 \%$ and $\Delta \varphi=5^{0}$. In the last case the change of RF field amplitude was no more then $10 \%$.

4. In order to improvement output beam parameters and reduce tolerances on amplitude and phase of RF field it is prefer RF power system with RF supplies which power is increased step by step. The proposed correction method will be efficient if RF supply have powered equal and small cavity number (for example, 4-6).

\section{REFERENCES}

[1] M Hunmg, S N Simrock, "Requirements for the RF Control of the Vector Sum for Superconducting Proton Linacs“, DESY, Notkestr 85, D-22603 Hamburg, Germany $\mathrm{T}$ Schilcher, PSI, CH-5232 Villigen, Switzerland. 